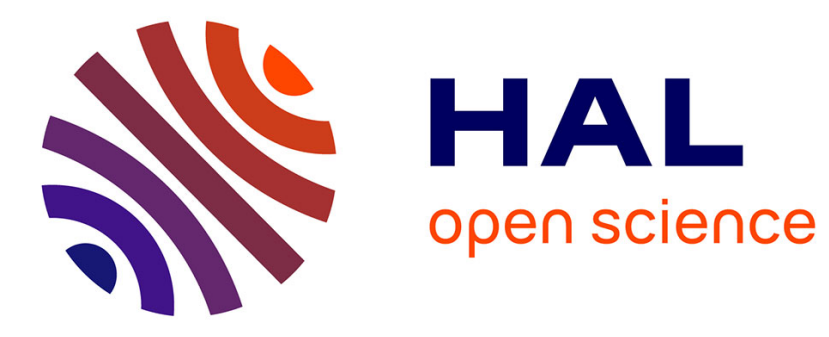

\title{
Morphology-based cerebrovascular atlas
}

Alice Dufour, Christian Ronse, Joseph Baruthio, Olena Tankyevych, Hugues Talbot, Nicolas Passat

\section{To cite this version:}

Alice Dufour, Christian Ronse, Joseph Baruthio, Olena Tankyevych, Hugues Talbot, et al.. Morphology-based cerebrovascular atlas. International Symposium on Biomedical Imaging (ISBI), 2013, San-Francisco, CA, United States. pp.1210-1214, 10.1109/ISBI.2013.6556698 • hal-00865887

\section{HAL Id: hal-00865887 https://hal.science/hal-00865887}

Submitted on 25 Sep 2013

HAL is a multi-disciplinary open access archive for the deposit and dissemination of scientific research documents, whether they are published or not. The documents may come from teaching and research institutions in France or abroad, or from public or private research centers.
L'archive ouverte pluridisciplinaire HAL, est destinée au dépôt et à la diffusion de documents scientifiques de niveau recherche, publiés ou non, émanant des établissements d'enseignement et de recherche français ou étrangers, des laboratoires publics ou privés. 


\title{
MORPHOLOGY-BASED CEREBROVASCULAR ATLAS
}

\author{
A. Dufour, C. Ronse, J. Baruthio ${ }^{(a)}$ \\ O. Tankyevych ${ }^{(b)}$ \\ H. Talbot ${ }^{(c)}$ \\ N. Passat ${ }^{(d)}$
}

(a) ICube, UMR CNRS, Université de Strasbourg, France

(b) LISSI, EA 3956, Université Paris-Est, France

(c) LIGM, UMR CNRS 8049, Université Paris-Est, France

(d) CReSTIC, EA 3804, Université de Reims, France

\begin{abstract}
Cerebrovascular atlases can be used to improve medical tasks requiring the analysis of 3D angiographic data. The generation of such atlases remains however a complex and infrequently considered issue. The existing approaches rely on information exclusively related to the vessels. We alternatively investigate a new way, consisting of using both vascular and morphological information (i.e., cerebral structures) to improve the accuracy and relevance of the obtained vascular atlases. Experiments emphasize improvements in the main steps of the atlas generation process impacted by the use of morphological information. An example of cerebrovascular atlas obtained from a dataset of 56 MRAs acquired from several acquisition devices is finally provided.
\end{abstract}

Index Terms - Vascular atlas, brain atlas, magnetic resonance angiography, spatial relationship.

\section{INTRODUCTION}

3D angiographic imaging, and in particular magnetic resonance angiography (MRA), provides solutions for noninvasive and non-irradiant exploration of cerebrovascular networks. The specificities of MRA data (sparseness, low SNR, artifacts), together with their usefulness in crucial issues (detection, quantification and follow-up of vascular pathologies) have motivated the development of specific image analysis techniques devoted to vascular structures [1].

In this context, the design of cerebrovascular atlases [2], and in particular statistical ones [3], provides solutions for several applications including, e.g., vessel segmentation, identification of vascular abnormalities, or vessel labeling.

Contrary to most of vascular networks (e.g., coronary arteries [4], that show a simple geometrical and topological structure), the cerebrovasculature presents challenging specificities for atlas generation purposes. The complexity of its topology, the closeness and parallel paths of veins and arteries, their tortuosity, or the heterogeneity of their size, all make cerebrovascular model generation a difficult task.

This work has been funded by a PhD grant of the Région Alsace (France) and the Centre National de la Recherche Scientifique (CNRS, France).
Very few works have been devoted to statistical cerebrovascular atlases (able to handle interindividual variability). Among them, the ones leading to the richest atlases [3] provide information about vascular presence probability, vessels size and orientation. Such as their predecessors [5, 6], they only rely on vascular information directly gathered from angiographic data. In particular, they do not take advantage of the information provided by the relations between vessels and the neighboring cerebral structures.

In this article, we improve the cerebrovascular atlas generation process developed in [3] by using both vascular and morphological information, carried out by MRA and standard MRI data, respectively. To reach that goal, we introduce a brain atlas as additional input of the process. We then use the supplementary anatomical information, in particular about the relations between vessels and cerebral structures, to improve the accuracy and richness of the proposed methodology.

This article is organized as follows. Sec. 2 summarises the enriched atlas generation methodology, and its specificities w.r.t. [3]. Sec. 3 describes more precisely the improvements induced by the use of a morphological atlas. Sec. 4 provides experimental validations. An example of cerebrovascular atlas, such as perspective works, are proposed in Sec. 5.

\section{OUTLINE OF THE METHODOLOGY}

The pipeline proposed in [3] took as input $k$ 3D angiographic images $A_{i}: \Omega \rightarrow V$, and $k$ morphological images $M_{i}: \Omega \rightarrow$ $V$, each $M_{i}$ being associated to $A_{i}$. (The pairs $\left(M_{i}, A_{i}\right)$ were chosen as the magnitude and phase images of phase-contrast (PC) MRAs.) It relied on four successive steps: (i) vessel segmentation; (ii) skeletonisation and size evaluation; (iii) registration; and (iv) information fusion. It provided as output an arteriovenous atlas $\mathcal{A}$, modelling statistical information: vascular probability density; vessels size and absolute orientation. This atlas was mapped on a mean morphological image $\mathcal{M}: \Omega \rightarrow V$ obtained from the registered images $M_{i}$. In this former protocol, the sole use of morphological information lied in the registration step (iii), performed on the $M_{i}$, to avoid the ill-posed problem of angiographic image registration [7]. 

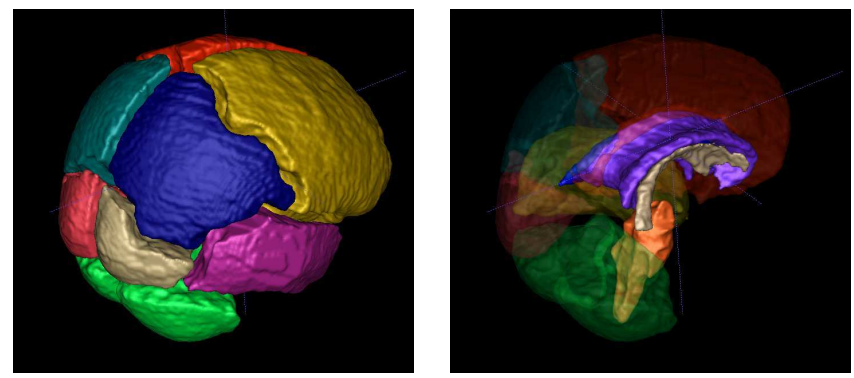

Fig. 1. 3D visualization of the morphological (brain) atlas $\mathcal{B}$ used as input of the proposed pipeline (see Sec. 3.1).

The proposed new pipeline more strongly relies on morphological information. Indeed, we consider as additional input a morphological atlas $\mathcal{B}$, that models the main brain structures presenting spatial relations with the vascular ones observed in MRA data. The successive steps of this new pipeline are then the following ones:

(i) Segmentation: Arterial $\left(a_{i}\right)$ and venous $\left(v_{i}\right) 3 \mathrm{D}$ volumes are extracted from the $A_{i}$ images.

(ii) Skeletonisation: 1D vessel centerlines $\left(a_{i}^{s}, v_{i}^{s}\right)$ are extracted from $\left(a_{i}, v_{i}\right)$ volumes.

(iii) Differential analysis: A multiscale Hessian-based analysis is applied on the $A_{i}$ images to determine the vessels size $\left(s_{i}\right)$ and orientation $\left(o_{i}\right)$.

(iv) (Co)registration: A deformation field $\mathcal{D}_{i}: \Omega \rightarrow \Omega$ is computed for each $A_{i}$ (based on $M_{i}$ ), in order to map the gathered information into a unique spatial reference.

(v) Atlas formation: The $a_{i}, v_{i}, s_{i}$ and $o_{i}$ are fused (with respect to $\mathcal{D}_{i}$ ) to finally lead to the vascular atlas $\mathcal{A}$.

Note that each of these generic steps can be instantiated by any (segmentation, registration, etc.) method using/providing adequate inputs and outputs. This new pipeline (see Fig. 5, in Appendix) leads to enriched vascular atlases

$$
\begin{aligned}
\mathcal{A}: \Omega & \rightarrow \mathbb{P} \times \mathbb{P} \times \mathbb{P} \times \mathbb{S} \times \mathbb{T} \\
x & \mapsto(\delta, \alpha, \tau, \sigma, \omega)
\end{aligned}
$$

where $\mathbb{P}=[0,1]$ and $\mathbb{S}=\mathbb{R}^{+} \times \mathbb{R}^{+}$are probability and mean value/standard deviation information, and $\mathbb{T}$ is a tensor space. It provides, for each $x \in \Omega$ of an anatomical reference $(\mathcal{M})$ : vascular presence $(\delta)$; vein/artery $(\alpha)$ and tubular/junction $(\tau)$ discrimination; vessel sizes $(\sigma)$ and relative orientations $(\omega)$.

This pipeline presents novelties w.r.t. [3]: (i) the estimation of vessels size and orientation does no longer rely on segmented/skeletonised data, but from the images $A_{i}$, via Hessian analysis [8] performed at $a_{i}^{s}, v_{i}^{s}$, and are thus robust to segmentation errors; (ii) deformation fields used to fuse morphological information of the $M_{i}$ images are now obtained by a coregistration process [9], then avoiding to use a given $M_{i}$ as (possibly biased) reference; (iii) fusion of vessel orientations is now performed by using translation, but also rotation information, provided by the $3 \mathrm{D}$ deformation fields, leading to more accurate relative orientations, instead of absolute ones.

In addition to these improvements -deriving from progress, e.g., in registration or filtering- some other ones, strongly linked to the relationships between vascular and morphological structures, have been obtained by involving a brain atlas in the process, as discussed in the sequel.

\section{MORPHOLOGICAL APPROACH FOR CEREBROVASCULAR ATLAS GENERATION}

\subsection{Morphological brain atlas}

The brain atlas considered in this work models morphological structures involved in the determination of specific vessel properties. In particular, it divides the intracranial volume into 13 regions (Fig. 1): brain hemispheres (divided into lobes), cerebellum, brain stem, ventricles and corpus callosum. Indeed, vessels visualized in MRA data (of size larger than $0.5 \mathrm{~mm}$ ) do not intersect these regions, but generally remain adjacent to the associated morphological structures.

This atlas was created by combining information provided by two state-of-the-art statistical atlases, namely the MNI152 atlas [10] and the Harvard-Oxford atlas [11]. The regions of interest were processed by mathematical morphology operators (not described here due to lack of space). In particular, the fuzzy regions provided by the two native atlases were turned into binary ones, separated by gaps, thus authorizing remaining space for vessel positioning (generally not considered in standard morphological atlases) and for fuzzy relationships processing, related to some of the steps of the pipeline.

\subsection{Morphology-based methodological improvements}

\subsubsection{Spatial guidance of segmentation and labelling}

While vessels were segmented as a whole in [3], we now propose to discriminate veins from arteries. This can be done by using a competitive seeded region-growing (in our case, based on grey-scale connectedness [12]). In this context, the usefulness of $\mathcal{B}$ is twofold: (i) the determination of seeds can be automated by the localization of specific arterious or venous loci (see Sec. 3.2.2); (ii) the grey-scale connectedness associated to $A_{i}$ images can be enriched by the use of $\mathcal{B}$. In particular, by labeling the brain structures as non-vascular areas, thus forbidding therein the by-pass of vessels, grey-scale connectedness can by involved in a geodesic approach, thus improving the robustness of vessel detection.

\subsubsection{Determination of landmarks and blood flow direction}

As stated above, $\mathcal{B}$ can be used to identify vascular landmarks. The modelling of spatial relationships between brain regions and neighbouring vessels can in particular be performed thanks to fuzzy mathematical models [13] (e.g., "in direction of", "between", "close to", "above"). Besides the 
only determination of such landmarks, these spatial relationships can be efficiently involved in two tasks: $(i)$ the characterisation of junctions/bifurcations areas (e.g., Willis polygon, confluens sinuum, etc.) vs. the remaining "tubular" parts of the vascular networks (this can guide the further computation of vessel orientations, that can be restricted to the tubular regions, and further propagated); (ii) the information provided by $\mathcal{B}$ also enable the determination of blood flow direction. This information is of precious use to enrich the orientation fields, provided by Hessian analysis, into vector fields, that can unambiguously be averaged during the final fusion step.

\subsubsection{Spatial guidance of anisotropic information fusion}

Once correctly registered (based on $\mathcal{D}_{i}$ ), the information computed for each $A_{i}$ must be fused into a single entity. Large vessels -which present low variability- are then globally superimposed, and their averaging is globally straightforward. In contrast, for smaller vessels -which present higher variability- the registration step leads, in general, to unsatisfactory spatial correlations. To deal with the induced sparseness, it is then required to propagate the information carried out by such vessels in their neighborhood. In particular, this can be done by Gaussian diffusion. However, since isotropic diffusion may lead to spreading of vascular information in non-relevant areas, the considered kernels should be designed in accordance to the anatomical structures. Based on the hypothesis that the vessels are located along brain structure surfaces, the considered kernels are then defined as "geodesic" ellipsoids (mostly, oblate spheroids parallel to these surfaces), thus leading to more accurate results.

\section{EXPERIMENTAL VALIDATIONS}

In this section, we experimentally validate some of the most crucial steps supposed to be improved by the morphologybased approach. These validations are completed by more qualitative (and subjective) ones, in Fig. 6 (in Appendix).

\subsection{Vein/artery segmentation and labelling}

In order to validate the morphology-based strategy considered for vein/artery segmentation, we have carried out the proposed method (Sec. 3.2.1) with and without geodesic constraints related to $\mathcal{B}$. This was done on two (low resolution PC, and high resolution time-of-flight (TOF)) MRAs for which ground-truth was provided by a medical expert. The chosen seeds were the Willis polygon and confluens sinuum, for arteries and veins, respectively. It has been experimentally observed that the size of these seeds did not actually influence the results; this size was then set to XX voxels. Fig. 2(a) compares the geodesic/non-geodesic version of the method in terms of global vessel segmentation. Fig. 2(b) focuses on the vein/artery discrimination errors inside the true positives,

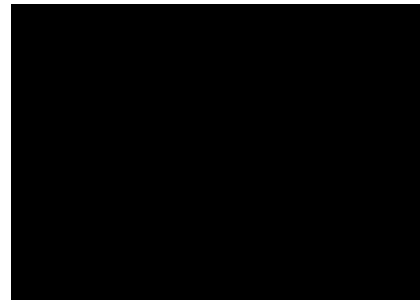

(a)

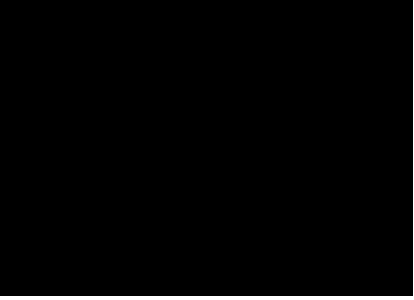

(b)
Fig. 2. Segmentation and labelling with/without geodesic constraints. (a) ROC curves for vessel segmentation. (b) Vein/artery labelling error ratio according to the true positive computed fraction.

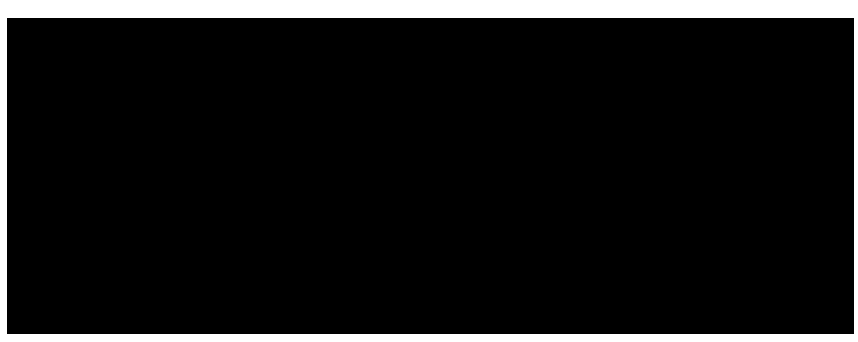

Fig. 3. Comparison of vessel orientations spreading, according to the vessel sizes.

during their computation from the seeds. These results emphasize the better accuracy of the geodesic approach.

\subsection{Vessel/vascular flow orientations}

As stated in Sec. 3.2.2, the supplementary information providing blood flow direction enables to fuse information about vessel orientation without ambiguity (by opposition to [3], where the fusion of two orthogonal direction was an ill-posed problem). The accuracy of the obtained result, can be assessed in particular w.r.t. the spreading of orientations over their mean values. In particular, this spreading should be low for larger vessels (which are globally invariant). Following this paradigm, the orientations obtained with the proposed methodology have been compared with those obtained from [3]. Fig. 3 tends to show that the orientations are now better modeled than in [3]. This analysis provides indirect clues of the relevance of methodological novelties related, e.g., to Hessian analysis, anisotropic diffusion and registration.

To better emphasise the relative importance of these methodological novelties, complementary validations are currently in progress. They consist of testing independently each part of the process on synthetic data, for which the expected results are known a priori. The first results of these validations (that will be exhaustively presented in an extended version of this article) confirm those provided here on real data. 


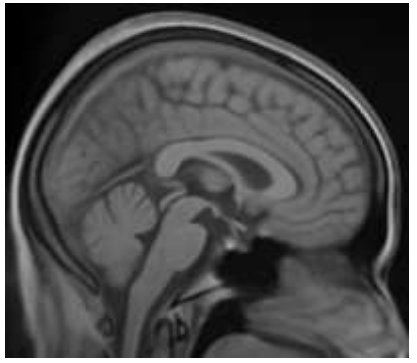

(a)

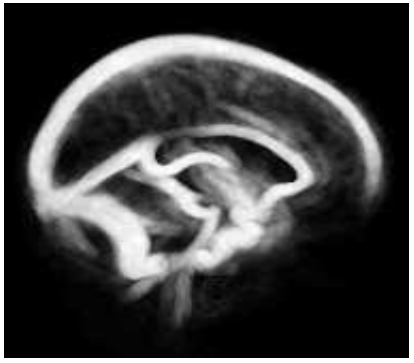

(b)

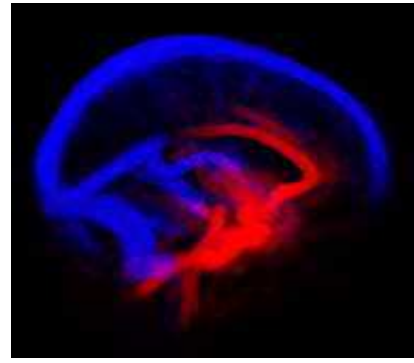

(c)

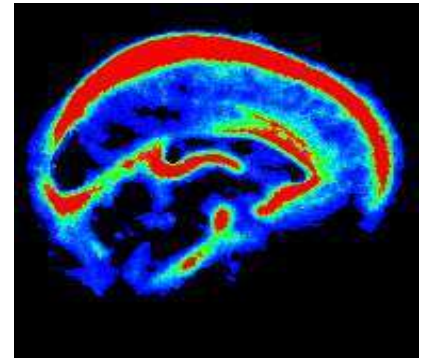

(d)

Fig. 4. Vascular atlas $\mathcal{A}$ (b-d, MIP visualisation) and its morphological reference $\mathcal{M}$ (a, sagittal view). (b) Vessel probability density $\delta$. (c) Vein/artery probability $\alpha$. (d) Vessel sizes (d).

\section{RESULTS}

In order to qualitatively illustrate the proposed methodology, it was applied on a dataset of 56 MRA images $\left(A_{i}\right)$, including both (millimetric) PC and (submillimetric) TOF MRAs. Concerning the $M_{i}$ images, the PC (phase) MRA were associated to their magnitude image, while the TOF MRA were associated to T1 MRI acquired during the same exam. The computed atlas is illustrated in Fig. 4 (see Fig. 7, in Appendix, for complementary illustrations).

Based on these results, further works will consist of embedding symbolic models of cerebrovasculature in the current statistical atlases, in order to lead to more complete vascular models, with perspectives in vessel labeling, e.g., for computer aided diagnosis of cerebrovascular malformations.

\section{REFERENCES}

[1] O. Tankyevych, H. Talbot, N. Passat, M. Musacchio, and M. Lagneau, "Angiographic image analysis," in Medical Image Processing: Techniques and Applications, chapter 6, pp. 115-144. Springer, 2011.

[2] W. Nowinski, I. Volkau, Y. Marchenko, A. Thirunavuukarasuu, T. Ng, and V. Runge, "A 3D model of human cerebrovasculature derived from 3T magnetic resonance angiography," NeuroInformatics, vol. 7, pp. 23-36, 2009.

[3] N. Passat, C. Ronse, J. Baruthio, J.-P. Armspach, and C. Maillot, "Magnetic resonance angiography: From anatomical knowledge modeling to vessel segmentation," Medical Image Analysis, vol. 10, pp. 259-274, 2006.

[4] C. Lorenz and J. von Berg, "A comprehensive shape model of the heart," Medical Image Analysis, vol. 10, pp. 657-670, 2006.

[5] D. Cool, D. Chillet, J. Kim, J.-P. Guyon, M. Foskey, and S. R. Aylward, "Tissue-based affine registration of brain images to form a vascular density atlas," in MICCAI, 2003, pp. 9-15.

[6] B. Naegel, C. Ronse, and L. Soler, "Using grey-scale hit-or-miss transform for segmenting the portal network of the liver," in ISMM, 2005, pp. 429-440.

[7] S. R. Aylward, J. Jomier, S. Weeks, and E. Bullitt, "Registration and analysis of vascular images," International Journal of Computer Vision, vol. 55, pp. 123-138, 2003.

[8] Y. Sato, S. Nakajima, N. Shiraga, H. Atsumi, S. Yoshida, T. Koller, G. Gerig, and R. Kikinis, "Three-dimensional multi-scale line filter for segmentation and visualization of curvilinear structures in medical images," Medical Image Analysis, vol. 2, pp. 143-168, 1998.

[9] V. Noblet, C. Heinrich, F. Heitz, and J.-P. Armspach, "An efficient incremental strategy for constrained groupwise registration based on symmetric pairwise registration," Pattern Recognition Letters, vol. 33, pp. 283-290, 2012.

[10] D. L. Collins, C. J. Holmes, and T. M. Peters, "Automatic 3-D model-based neuroanatomical segmentation," Human Brain Mapping, vol. 3, pp. 190-208, 1995.

[11] R. S. Desikan, F. Ségonne, B. Fischl, B. T. Quinn, B. C. Dickerson, D. Blacker, R. L. Buckner, A. M. Dale, R. P. Maguire, B. T. Hyman, M. S. Albert, and R. J. Killiany, "An automated labeling system for subdividing the human cerebral cortex on MRI scans into gyral based regions of interest," NeuroImage, vol. 31, pp. 968-980, 2006.

[12] X. Tizon and Ö. Smedby, "Segmentation with grayscale connectedness can separate arteries and veins in MRA," Journal of Magnetic Resonance Imaging, vol. 15, pp. 438-445, 2002.

[13] I. Bloch, "Fuzzy spatial relationships for image processing and interpretation: A review," Image and Vision Computing, vol. 23, pp. 89-110, 2005. 


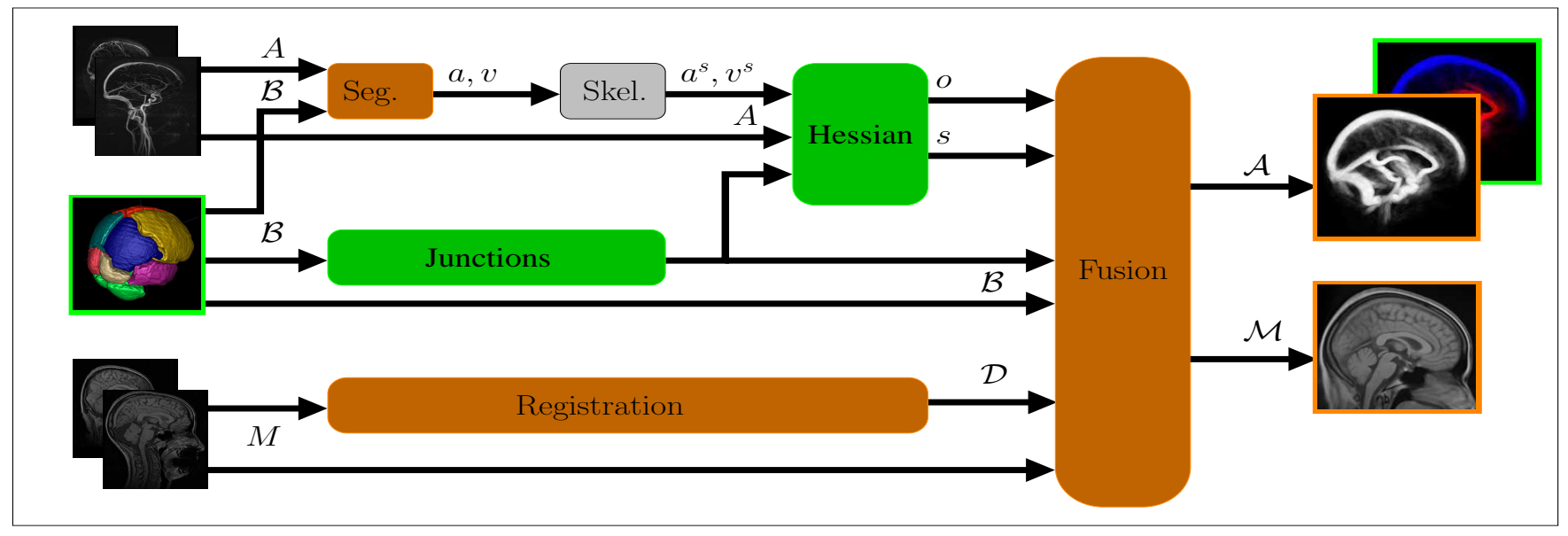

Fig. 5. Morphology-guided atlas generation pipeline (see Secs. 2 and 3.2). In green: new inputs and new steps. In orange: modified/improved steps.

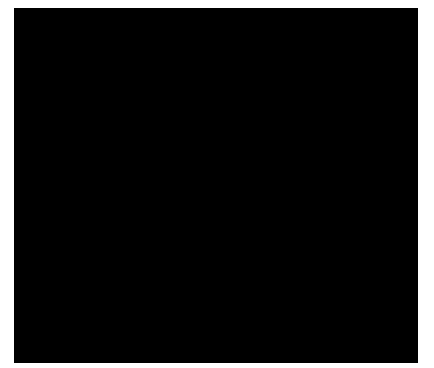

(a)

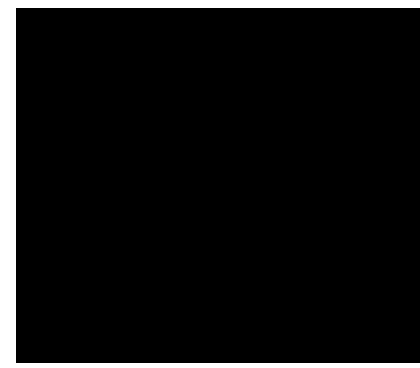

(b)

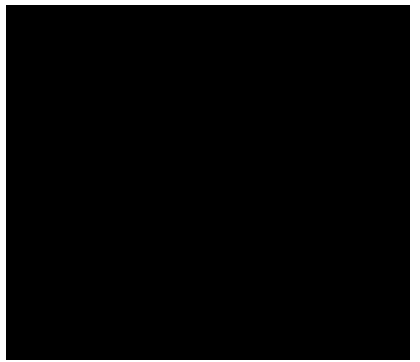

(c)

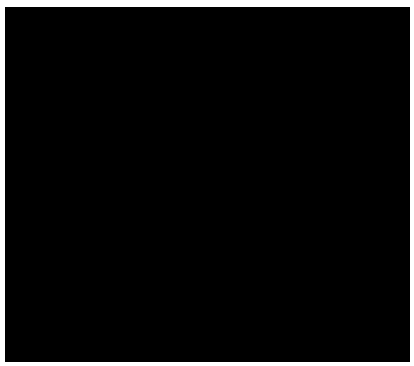

(d)

Fig. 6. (a,b) Effects of the (an)isotropic diffusion on the information fusion, illustrated on the arterial part of the vascular density field (coronal view, MIP visualisation): (a) isotropic and (b) anisotropic diffusion. (c,d) Orientation spreading (from low, in green, to high, in red) illustrated in the venous part of the atlas (sagittal slice): (c) with skeleton-based orientation and translation-based registration [3], and (d) with Hessian-based analysis and translation/rotation-based registration.

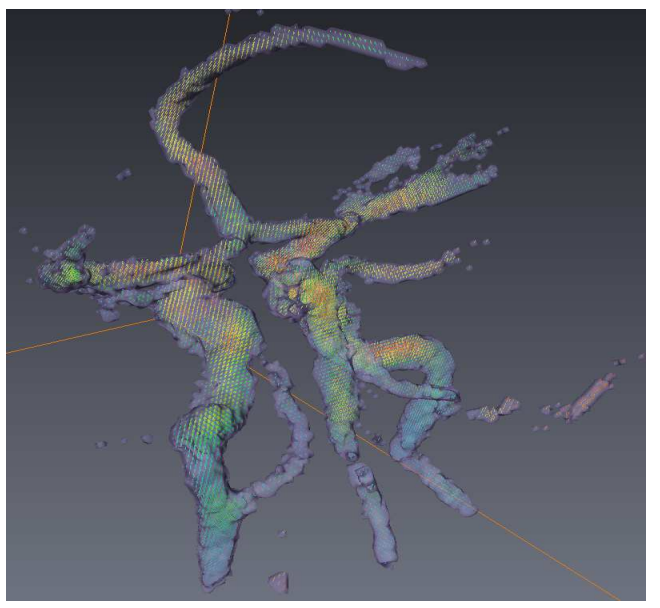

(a)

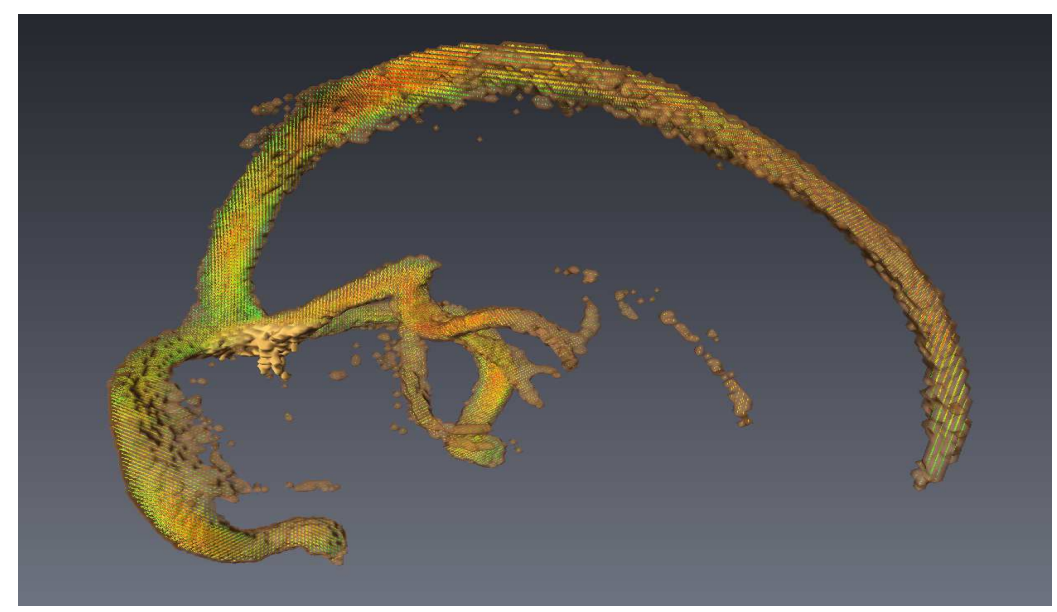

(b)

Fig. 7. $3 \mathrm{D}$ visualization of vessel orientations in the atlas $\mathcal{A}$ (see Sec. 5): arteries (a) and veins (b). The orientations are represented by vector fields, with additional color encoding in the axial (a) and sagittal plane (b). 